\title{
A Novel Alternately Energy Source Strategy for Re- Ablation of Patients with Recurrent Atrial Fibrillation - Cryoballoon or Radiofrequency Current Energy Ablation. A Case-Control Study
}

\author{
Qinghui Tang \\ Chinese Academy of Medical Sciences and Peking Union Medical College \\ XiaoGang Guo \\ Chinese Academy of Medical Sciences and Peking Union Medical College \\ Jian Ma ( $\triangle$ majian_fuwai@126.com ) \\ Chinese Academy of Medical Sciences and Peking Union Medical College
}

\section{Research Article}

Keywords: AF Recurrence, Repeat Ablation, Cryoballoon, Radiofrequency Ablation, Alternate energy sequence ablation strategy

Posted Date: December 22nd, 2021

DOI: https://doi.org/10.21203/rs.3.rs-1165778/v1

License: (c) (i) This work is licensed under a Creative Commons Attribution 4.0 International License.

Read Full License 


\section{Abstract}

Background: Atrial fibrillation recurrence after circumferential pulmonary vein (PV) isolation was common. Which ablation technique is better for repeat ablation in patients with recurrent atrial fibrillation (AF) remains unclear. We aimed to investigate long-term efficacy of repeat ablation using a novel alternately energy source sequence for re-ablation of patients with recurrent atrial fibrillation: cryoballoon (CB) re-ablation for patients with a failed radiofrequency (RFC) ablation (RFC-CB redo group); radiofrequency energy re- ablation for patients with a failed cryoballoon ablation (CB-RFC redo group).

Method: Recurrent AF patients received a repeat ablation procedure in our hospital were enrolled into the study. Demographic and re-ablation procedural characteristics and outcomes were compared among groups.

Results: A total of 156 patients were enrolled into the study, 60 patients (38.5\%) were in the CB-RFC-redo group and 96 patients (61.5\%) were in the RFC-CB-redo group. Longer duration of AF (69.31 $\pm 64.69 \mathrm{vs}$ $50.78 \pm 51.48$ months; $P=0.039)$ and longer time from first ablation to re-ablation $(54.02 \pm 38.10 \mathrm{vs}$ $14.2 \pm 10.5$ months; $P=0.001$ ) were observed in the RFC-CB-redo group as compared with the CB-RFC redo group. Early recurrence rates of atrial fibrillation following initial ablation were equal among groups (RFCCB-redo group: $42.7 \%$ vs CB-RFC-redo group:48.3\% , $\mathrm{p}=0.515)$. The number of reconnected PVs was significantly higher in the RFC-CB redo group than the CB-RFC redo group ( $3.36 \pm 0.96$ vs $1.50 \pm 0.81$, $p=0.01$ ). During the average follow-up of $10.7 \pm 2.41$ months, significantly less $A F$ recurrence was observed in the CB-RFC redo group ( $16.7 \%$ vs $31.3 \%, p=0.045)$. In the multivariate analysis, different energy ablation sequence, AF type and early recurrence after the initial ablation were independent predictors of AF recurrence after re-ablation.

Conclusions: Alternate energy source sequence strategy for re-ablation of patients with recurrent atrial fibrillation was safe and moderately effective. However, Large multi-center studies must be warranted to provide conclusive evidence.

\section{Background}

Atrial fibrillation (AF) is the most common clinical arrhythmia in the world, which often impaired life quality and leaded to serious complications [1]. Radiofrequency catheter (RFC) ablation or cryoballoon (CB) ablation therapy based on pulmonary vein (PV) isolation is an well-established treatment strategy for drug-refractory AF [2-3]. Long lasting PVs isolation is fundamental to avoid the AF recurrence and is the primary goal of AF ablation regardless of RFC or CB ablation [3-4]. Despite the high success rate of RFC or CB ablation, $20-30 \%$ of AF patients may suffer from PVs-left atrium(LA) reconnection and AF recurrence [5]. Due to the high AF recurrence rate after RFC ablation or $\mathrm{CB}$ ablation, repeated ablation is very common in the clinical practice related to recurrent AF patients [6-7]. Pulmonary vein (PV)-LA reconnection is the main cause of recurrent arrhythmia after RFC ablation or CB ablation [8-9]. However, appropriate AF ablation strategy (RFC or CB ablation) used in the repeat ablation is still controversial. 
Recent, repeat $A F$ ablation using the alternately energy source sequence ( $C B$ re-ablation after a failed RFC ablation, RFC repeat ablation following a failed CB ablation) was suggested [9]. However, there was still few data comparing the long-term efficacy or safety of this alternately energy source ablation strategy for recurrent AF patients because only one study reported the strategy [9]. Therefore, the present study was aimed to compare the electrophysiological outcome of those patients who underwent repeat ablation and investigate long-term efficacy and safety of alternate energy source ablation strategy (Cryoballoon or Radiofrequency Ablation) for repeat ablation procedure in patients with recurrent atrial fibrillation.

\section{Methods}

\section{Patients}

We retrospectively recruited 250 patients from September 2016 to February 2018, all patients who underwent a repeat catheter ablation procedure after a failed RFC ablation or CB PV isolation were eligible in this study. The study protocol was approved by the Human Research Ethics Committee of the Fuwai hospital according to the Helsinki Declaration. Repeat ablation strategy was used by the alternately energy source ablation sequence. For instance, patients firstly ablated with RFC ablation underwent re-ablation with $\mathrm{CB}$ (RFC-CB-redo group) and patients initially treated with $\mathrm{CB}$ ablation underwent redo PV isolation with RFC ablation (CB- RFC-redo group). All patients provided written informed consent.

\section{Radiofrequency ablation procedure}

The ablation procedure was performed on uninterrupted oral anticoagulant drug on the ablation procedural day. All the anti-arrhythmic drug except amiodarone were stopped five half-live time before the procedure. Vascular puncture was performed under local anesthesia. A 6F decapolar electrode catheter via the right internal jugular vein was inserted into the coronary sinus. Transseptal puncture was performed under X-fluoroscopy and the Lasso [Biosense Webster, Diamond Bar, CA, USA]) and 3.5-mm tip irrigated ablation catheter (SmartTouch catheter (Biosense Webster)) were advanced to the LA . LA map construction was performed using the CARTO 3 system (Biosense Webster). Each PV ostium was identified by selective venography and was then tagged on the LA electroanatomic map. The Lasso catheter [Biosense Webster, Diamond Bar, CA, USA]) was placed approximately $0.5 \mathrm{~cm}$ within the PV ostium for real-time monitoring of LA-PVs conduction. The infusion rate of perfusion during radiofrequency ablation is $17 \mathrm{ml} / \mathrm{min}$, the maximum temperature is $43^{\circ} \mathrm{C}$, and the contact force is $10-$ $20 \mathrm{~g}$ and each ablation point is at least $30 \mathrm{~s}$ to achieve more than $70 \%$ local voltage reduction. Circumferential PV ostium ablation to isolate the PV veins would be performed if LA-PVs conduction was demonstrated. If AF continued to persist after PVs isolation, the cardioversion was performed. 


\section{Second-generation cryoballoon ablation procedure}

Cryoballoon ablation procedure was performed under mild conscious sedation. Simply, a $28 \mathrm{~mm}$ secondgeneration cryoballoon catheter (Art Front Advance, Medtronic, Minneapolis, Mn, USA) and a 20-mmdiameter inner lumen Achieve catheter (Achieve ${ }^{\mathrm{TM}}$, Medtronic, MN, USA) were inserted through a $12 \mathrm{~F}$ steerable sheath (FlexCath, Medtronic, Minneapolis, Mn, USA) and then directed to each targeted PV ostium to record PV signals before, during and after ablation. The PV ostium occlusion was evaluated using the selective angiography via the inner lumen of the $\mathrm{CB}$ catheter. According to $\mathrm{CB}$ ablation routine, single ablation procedure time for second- generation balloon was $\leq 180 \mathrm{~s}$. If the PVs isolated were completed within $60 \mathrm{~s}$, only one CB ablation cycle was used. If PVs isolation was achieved and PVs-LA conduction did not recur, no bonus ablation was needed. If a time-to-isolation (TTI) $\geq 60 \mathrm{~s}$, a second CB cycle application within $180 \mathrm{~s}$ was performed. Phrenic nerve stimulation was applied through a steerable multipolar catheter in the superior vena cava (cycle length $1000 \mathrm{~ms}, 15 \mathrm{~mA} / 3.0 \mathrm{~ms}$ pulse width) during isolation of the right-sided PVs to reduce the risk of phrenic nerve palsy. If a decrease of phrenic nerve capture was observed, the CB ablation procedure was immediately stopped.

\section{Repeat Ablation Procedure}

During the repeat ablation procedure (regardless of RFC or CB ablation used), the first objective was to check the LA -PVs conduction using a multipolar circular catheter (Lasso ${ }^{\circledR}$ catheter, Biosense Webster or Achieve mapping catheter, Medtronic, Minneapolis, MN). LA- PVs reconnection was defined as an absence of entry block at the PV ostium during pacing [11]. Any repetitive premature atrial complex, atrial tachycardia, and beats triggering atrial flutter or AF were identified as the non-PVs triggers and were targeted for re-ablation. If the patients in the initial ablation procedure originally treated with RFC ablation, those patients would be re-ablated with $\mathrm{CB}$ ablation. By contrary, the patients originally treated with $\mathrm{CB}$ ablation, RFC ablation would be applied at the redo-procedure.

\section{Follow-up}

Post re-ablation follow-up protocol was for all patients, the data was collected during outpatient visits, or from all other forms of contact with the patients or their referring doctors, including clinical letters and telephone calls. Clinical follow-up and 12 lead ECG were arranged at 3, 6 and 12 months after ablation, and then once a year. For each patient with recurrent arrhythmias and patients with serious symptoms, more frequent follow-up should be arranged as patients needed. Any episodes of atrial tachyarrhythmia of longer than $30 \mathrm{~s}$ as detected by the ECG or Holter beyond the black 3-month period were considered as $\mathrm{AF}$ recurrence.

\section{Statistical analysis}


Continuous variables are expressed as Mean \pm SD, while classified variables are expressed as percentage. The survival curve was analyzed by the Kaplan Meier method. The Cox regression analysis was used to analyze the risk predictors of AF recurrence. Nonparametric $t$ tests were used to examine continuous outcomes and Pearson's chi-squared test was used to compare categorical outcomes among groups. A P-value of $<0.05$ was considered statistically significant. All statistics were performed using the SPSS version 25.0 (SPSS Inc, Chicago, IL, USA).

\section{Results}

\section{Baseline characteristics}

Among the 250 consecutive patients with AF recurrence who underwent repeat ablation, 85 patients who met the major exclusion criteria and 9 patients lost to follow-up were excluded (as was shown in Fig.1). The Research flow chart and redo-ablation procedure were summarized in Fig.1. A total of 156 patients with a repeat PVs isolation procedure in our hospital were enrolled into the study, 60 patients $(38.5 \%)$ were in the CB-RFC-redo group and 96 patients (61.5\%) were in the RFC-CB-redo group. Patient characteristics were shown in Table 1. A total of 34 patients $(21.8 \%)$ had persistent AF, while 122 patients (78.2\%) had paroxysmal AF (44 patients were in CB-RFC-redo group and 78 patients were in RFC-CB-redo group). Mean age of enrolled patients was $58.19 \pm 9.26$ years, 114 patients $(73.1 \%)$ were male, and the mean Body Mass Index (BMI) was $25.9 \pm 2.9 \mathrm{~kg} / \mathrm{m}^{2}$. The average $\mathrm{CHA}_{2} \mathrm{DS}_{2}-\mathrm{VASc}$ score was not statistically significant difference $(1.02 \pm 1.01$ in the CB-RFC-redo group vs $1.29 \pm 1.09$ in the RFC-CB-redo group, $p=0.249)$. Similarly, the average HAS-BLED score of two group was not statistically significant difference $(0.31 \pm 0.47$ in the CB-RFC-redo group vs $0.72 \pm 0.71$ in the RFC-CB-redo group, $p=0.090)$. The mean LA diameter at the time of enrollment in this study was $38.85 \pm 4.60 \mathrm{~mm}$. The two groups were comparable in terms of age, BMI, AF history, drug usage and cardiac geometry. Patients in the RFC-CBredo group had longer duration of AF history ( $69.31 \pm 64.69$ vs $50.78 \pm 51.48$ months, $P=0.039)$. Early recurrence(ER) rate after the first ablation procedure in the CB-RFC-redo group and RFC-CB-redo group was not significant $(48.3 \%$ vs $42.7 \%, p=0.515)$. Similarly, no significant difference was found for the other clinical baseline features of the two groups (Table 1). Most re-ablation patients recurred with paroxysmal AF $(67 / 96,69.8 \%$ patients in the RFC-CB redo group vs $44 / 60,73.3 \%$ patients in the CB-RFC redo group, $p=0.15), 34$ patients recurred with persistent atrial fibrillation $(18 / 96,18.8 \%$ patients in the RFC-CB redo group vs 16/60, 26.7\%patients in the CB-RFC redo group, $p=0.35), 24$ patients recurred with atrial tachycardia or atrial flutter $(11 / 96,11.5 \%$ patients in the RFC-CB redo group vs $13 / 60,21.7 \%$ patients in the CB-RFC redo group, $p=0.035$ ) (Fig. 2). As was indicated in Fig 2, more patients recurred with atrial tachycardia or atrial flutter were observed in those patients after initial CB ablation.

\section{Electrophysiological Findings}

The mean time from initial ablation to repeat ablation was longer in the RFC-CB redo group than those in the CB-RFC redo group ( $54.02 \pm 38.10$ vs $14.2 \pm 10.5$ months, respectively; $P=0.001$ ). During the redo 
ablation procedure, 148 (94.9\%) patients were found to have reconnected left atrial-PVs conduction, while 7 patients in CB-RFC-redo group and one patients in RFC-CB-redo group had none reconnected PVs at the time of repeat ablation ( $p=0.045$ ) (Table 2). Overall, the mean number of reconnected LA-PVs per patient was significantly higher in the RFC-CB redo group $(3.36 \pm 0.96)$ than in the CB-RFC-redo group $(1.50 \pm$ $0.81, p=0.010$ ) ( Table 2). The number of reconnected LA-PVs per patient in both group is depicted in Fig. 3. As was shown in Fig. 3, 64 patients (66.7\%) in the RFC-CB redo group showed reconnected PVs requiring repeat ablation of all four $\mathrm{PVs}$, as compared with none patients in the CB-RFC-redo group $(p<0.001)$. In the CB-RFC-redo group, there were significantly more reconnected left superior PVs $(32 / 91$, $35.2 \%)$ and fewer reconnected right superior PVs (14/91, 15.4\%), in compared to other PVs. In the RFCCB-redo group, there were significantly more reconnected left superior PVs (89/317, 28.1\%), in compared to other PVs. More PVs treated per patient were observed in the RFC-CB-redo group, in comparison with CB-RFC-redo group (3.36 \pm 0.96 vs $1.5 \pm 0.81, p=0.01$ ) (Table 3 ). More non-PVs related atrial arrhythmia was observed in the initial CB ablation (CB-RFC-redo vs RFC-CB-redo: $11.7 \%$ vs $1.04 \%$, $\mathrm{p}=0.030$ ) at the re-ablation procedure (Table 2 ).

\section{Repeat ablation procedural Data}

Procedural data for repeat ablation was summarized in Table 4. The mean repeat ablation procedure time was $70.6 \pm 20.7 \mathrm{~min}$ in the CB-RFC-redo group, and it was significantly shorter in the RFC-CB-Redo group (49.5 $\pm 12.7 \mathrm{~min}, \mathrm{p}=0.025)$. However, patients in the RFC-CB redo group required longer fluoroscopy time $(15.9 \pm 0.8 \mathrm{~min}$ vs $4.2 \pm 0.9 \mathrm{~min}, \quad \mathrm{p}=0.001)$ and more fluoroscopy dose ( $232.5 \pm 138.1 \mathrm{mGy}$

vs $156.3 \pm 15.3 \mathrm{mGy}, \mathrm{p}=0.02$ ) in compared to CB-RFC redo group. More patients in the CB-RFC redo group need cavotricuspid isthmus ablation (27 patients vs 19

patients, $\mathrm{p}=0.03$ ). One patients in the CB-RFC-redo group had pericardial tamponade, two patients in the RFC-CB-redo group had hemoptysis and two patients in the RFC-CB-redo group demonstrated a shorttime vagal reflex. No other severe adverse events, including acute cerebrovascular event, major bleeding event, embolic complication, vascular complication, PV stenosis, or death were reported.

\section{Recurrence of AF and Predictors}

The average follow-up time after repeat ablation was $10.7 \pm 2.41$ months (range $3-12$ months) in the total population: $10.4 \pm 2.59$ months in the RFC-CB redo group and $11.2 \pm 2.04$ months in the CB-RFC redo group $(p=0.06)$. Forty patients experienced $A F$ recurrence during follow-up, including thirty patients (31.3\%) in the RFC-CB redo group and ten patients (16.7\%) in the CB-RFC redo group. As was shown in Fig. 4, the freedom from AF recurrence after repeat ablation was $83.35 \%$ in the CB-RFC-redo group and $68.7 \%$ in the RFC-CB redo group, respectively $(H R=4.035$, Log-rank $P=0.045)$. Univariate Cox analysis showed that early recurrence (ER) after first ablation ( $H R=2.527,95 \% \mathrm{Cl}: 1.296-4.927, \mathrm{P}=0.006)$ and the different energy ablation sequence (RFC- $C B$ redo group) ( $H R=2.528,95 \% \mathrm{Cl}: 1.175-5.439, \mathrm{P}=0.018)$ were risk factors for $\mathrm{AF}$ recurrence following the repeat ablation. On the multivariate Cox regression 
analysis, different energy source ablation sequence (RFC- CB redo group) was still independently risk factors for $A F$ recurrence following the repeat ablation $(H R=2.314,95 \% \mathrm{Cl}: 1.128-4.747, \mathrm{P}=0.022)$. Moreover, the multivariate Cox regression analysis also shown that AF type (persistent AF) and early recurrence $(E R)$ after the initial ablation were also significantly associated with the poor outcome after reablation $(\mathrm{HR}=2.107,95 \% \mathrm{Cl}: 1.085-4.091, \mathrm{P}=0.028 ; \mathrm{HR}=2.431,95 \% \mathrm{Cl}: 1.279-4.618, \mathrm{P}=0.007$, respectively)( Table 5). As was shown in Fig. 5A, those patients without early recurrence after the initial ablation had better re-ablation outcome compared with those with early recurrence regardless of ablation energy source used $(82.6 \%$ vs $64.3 \%, H R=6.57$, Log-rank $P=0.010)$. The AF-free rate of persistent AF was 61.8\% after one-year follow-up (vs paroxysmal AF 77.9\% , HR=10.15, 95\% Cl: 10.35-11.1, P = 0.05) (Fig. 5B) regardless of the energy source of re-ablation. No severe complications or embolic events occurred during the follow-up period.

\section{Discussion}

\section{Major findings}

In this analysis, we aimed to describe long-term follow-up outcome of AF patients undergoing two alternate energy source sequence ablation (RFC-CB redo ablation vs CB-RFC redo ablation) for either recurrent AF. The main findings of our research were as follows, including: 1$) 40$ patients $(25.6 \%$ of the total population) recurred after repeat ablation procedure at the mean follow-up of $10.7 \pm 2.41 \mathrm{months} ; 2)$ we found that significantly fewer reconnected PVs were observed in patients originally treated with the CB ablation than in patients originally treated with RFC ablation ( $1.50 \pm 0.81$ vs $3.36 \pm 0.96, p=0.01$ per patients) and more non-PVs related atrial arrhythmia (CB-RFC-redo vs RFC-CB-redo: $11.7 \%$ vs $1.04 \%$, $p=0.030$ ) following the initial $C B$ ablation was observed at the re-ablation procedure ;3) for recurrent $A F$ with post-ablation, repeat ablation with the alternate energy source sequence was effective and safe, with up to $74.4 \%$ arrhythmia-free patients in the total population during the follow up; 4 ) the early recurrence after the initial ablation strongly predicted the poor outcome of the re-ablation. 5) different alternately energy source sequence ( AF patients originally treated with RFC ablation, then repeated with CB ablation at the redo procedure, RFC-CB redo group) was the predictive risk factor for $\mathrm{AF}$ recurrence after redo ablation;

\section{PVs Reconnection at the re-ablation procedure}

It was proved that durable PVs isolation is fundamental to freedom from AF recurrence and is also primary goal of catheter ablation therapy [11]. In this study, we found that recurrent AF patients initially treated with $\mathrm{CB}$ ablation had significantly fewer all four PVs reconnection and few reconnected PVs per patient with the mean of $1.50 \pm 0.81 \mathrm{PVs}$, in comparison with AF patients firstly treated with RFC ablation. In the FIRE AND ICE trial, patients initially treated with the CB ablation had significantly fewer reconnected PVs and thus required fewer lesions at the re-ablation procedure [6]. Generally, CB ablation shown catheter stability in anatomically challenging locations, compared to RFC ablation [12]. For instance, 
significantly fewer reconnected left superior PVs were observed in the CB initial ablation group. However, AF patients initially treated with RFC ablation had more left superior PVs reconnection and bilateral reconnected pulmonary veins in our study. These data may reflect differences in catheter stability in those anatomical challenging locations. RFC ablation usually created contiguous and transmural ablation line [13], but may not be readily achieved along the myocardial ridge separating the left-sided PVs from the LA appendage [14-15]. By contrast, the myocardial ridge may not influence the ability of the cryoballoon to create durable lesions in this area [16].

\section{Biophysics of Radiofrequency ablation vs Cryoablation}

Radiofrequency ablation is to transform electromagnetic energy into thermal energy and created myocardial injury around the pulmonary vein antrum [17-18]. However, myocardial injury caused by radiofrequency ablation is usually related to coagulative necrosis deformation[19], hemorrhagic injury[20] and inflammatory cell infiltration [21]around injury tissue. When the edema tissues subsided, these gaps around the PV antrum would resume LA-PVs conduction [22]. It has been well proved that the variable anatomical characteristics of PVs may have an impact on the efficacy of radiofrequency ablation [23]. Anatomical studies found that the muscle sleeves of the lower wall of the superior pulmonary vein and the upper wall of the inferior pulmonary vein were the thickest and catheter in these lesions is unstable [24], so those sites were also common location for atrial -pulmonary vein potential recovery after RFC ablation [25]. Furthermore, center experience would influence the efficiency of radiofrequency ablation [26]. It was reported that center experience was independent risk factor of late AF recurrence related to RFC ablation $[26,27]$. In the present study, $66.7 \%$ patients in the RFC-CB redo group showed all four PVs reconnection requiring repeat ablation, while significant fewer patients in the CB-RFC redo group required four PVs ablation. Cryoablation emerged as a novel ablation technique improving the outcome of PVs isolation and then minimized thrombus formation [16], preserved the basic underlying tissue architecture and alleviated inflammation reaction [11]. Because the relapse of LA-PVs reconnection is still the main cause of late recurrent arrhythmia after the initial AF ablation with either CB or RFC ablation [8]. Cryoablation was usually with a durable PVs isolation [11]. However, more non-PVs triggers and LA flutters were found at repeat ablation following the CB ablation [11]. In this study, we found that 7 patients $(11.7 \%)$ firstly treated with CB ablation require non-PVs lesions ablation, while only one patient (1.04\%) following RFC ablation needed non-PVs lesions ablation.

\section{Alternately Ablation energy sequence and Recurrence of AF}

So far, there were few reports dedicated to evaluate the efficacy and safety of the usage of the alternately energy source sequence for repeat ablation of recurrent AF patients. At the average of $10.7 \pm 2.41$ months follow-up after repeat ablation, we found that a total of 40 patients $(25.6 \%)$ recurred after the re- ablation, with better re-ablation outcome in the CB-RFC redo group ( $16.7 \%$ vs $31.3 \%$, Log-rank $P=0.045)$. In Verlato $\mathrm{R}$ et al's study, significantly fewer AF recurrences (10.9\%) occurred in patients after RFC repeat ablation 
after initial CB PV isolation [9].The outcome of arrhythmia-free rate in this study was similar to previous published reports for repeat AF ablation (70\%-79.4\%) [9,27]. Consequently, the present study further supported the safety and modest efficacy of the alternately energy sequence for repeat ablation after RFC or $\mathrm{CB}$ ablation. Several factors had been established as potential risk predictors for the AF recurrence after re-ablation, such as the greater LA diameter, AF type, sex, obesity and $\mathrm{CHA}_{2} \mathrm{DS}_{2}$-VASc score. The Cox regression analysis indicated that the different energy source ablation sequence (RFC-CB redo group) was independently risk of $A F$ recurrence $(H R=2.528,95 \% \mathrm{Cl}: 1.175-5.439, \mathrm{P}=0.018)$. Conversely, RFC ablation after initial CB PV isolation (CB-RFC redo group) may be highly effective to prevent further AF recurrence following redo ablation procedure. Also, we found that the early AF recurrence in the initial ablation was association with the poor re-ablation outcome regardless of ablation energy used. The Kaplan-Meier analysis shown that those patients with early recurrence after the initial ablation had more AF recurrence compared with those without early recurrence $(35.7 \%$ vs $17.4 \%$, Log-rank $P=0.010)$ after re-ablation. A previous study by Miao et al [27] also reported that patients with early recurrences after initial radiofrequency ablation respond worse to repeat ablation, but initial ablation and re-ablation in their study were all performed by radiofrequency current energy. For other clinical risk factors, we found that LA diameter, sex and $\mathrm{CHA}_{2} \mathrm{DS}_{2}$-VASc score were not correlated with $\mathrm{AF}$ recurrence after re-ablation.

\section{Study limitations}

Several limitations existed in this study as follows: (1) this is a single-center retrospective study; Moreover, the sample of this study was small; Larger multi-center studies must be warranted to provide conclusive evidence; (2) In this study, 24-h Holter monitoring was used for follow-up investigate but it may be underpowered when implantable loop recorder was widely used now; (3) the AF history of some patients could not be precisely identified in the present study.

\section{Conclusions}

Alternately energy source sequence ablation strategy for re-ablation of patients with recurrent atrial fibrillation was effective and safe. However, Large multi-center randomized controlled trials must be warranted to provide conclusive evidence.

\section{Abbreviations}

AF『atrial fibrillation; BMI: body mass index; CAD: coronary heart disease; LVEF: left ventricular ejection fraction; LAD: Left atrial diameter; TIA: transient ischemic attack; RFC: radiofrequency current; CB: cryoballoon.

\section{Declarations}




\section{Acknowledgements}

The authors would like to thank the research staff for their help.

\section{Authors' contributions}

QHT and XGG: Conceptualization, Investigation, Data curation,

Methodology, Formal analysis, Validation, Writing original draft; JM: Conceptualization, Methodology, Validation, Formal analysis. All the authors were involved in the draft, revision and approval of the final version.

\section{Funding}

This study was supported by the National Natural Science Foundation of China (No.81670309).

\section{Availability of data and materials}

The datasets used and/or analyzed during the current study are available from the corresponding author on reasonable request.

\section{Ethics approval}

The retrospective study was approved by the Ethics Committee of the Fuwai Hospital, National Center for Cardiovascular Diseases, Chinese Academy of Medical Sciences and Peking Union Medical College ((No.2016-829) according to the Helsinki Declaration.

\section{Consent to participate}

Written informed consent was provided by all participants or their legal guardians. All methods were performed in accordance with the relevant guidelines and regulations of the Declaration of Helsinki.

\section{Consent for publication}

Not applicable.

\section{Competing Interests}


All authors had no conflicts of interest.

\section{References}

1. Heeringa J, van der Kuip DA, Hofman A, Kors JA, van Herpen G, Stricker BH, et al. Prevalence, incidence and lifetime risk of atrial fibrillation: the Rotterdam study. Eur Heart J. 2006;27:949-953.

2. Buist TJ, Adiyaman A, Smit JJJ, Ramdat Misier AR, Elvan A. Arrhythmia-free survival and pulmonary vein reconnection patterns after second-generation cryoballoon and contact-force radiofrequency pulmonary vein isolation. Clin Res Cardiol. 2018;107:498-506.

3. Ang R, Hunter RJ, Lim WY, Opel A, Ullah W, Providencia R, et al. Long term outcome and pulmonary vein reconnection of patients undergoing cryoablation and/or radiofrequency ablation: results from the Cryo Versus RF trial. J Atr Fibrillation. 2018;11(3):2072.

4. Ciconte G, Velagić V, Mugnai G, Saitoh Y, Irfan G, Hunuk B, et al. Electrophysiological findings following pulmonary vein isolation using radiofrequency catheter guided by contact-force and second generation cryoballoon: lessons from repeat ablation procedures. Europace. 2016;18:71-77.

5. Kuck KH, Brugada J, Fürnkranz A, Metzner A, Ouyang F, Chun KR, et al. FIRE AND ICE Investigators. Cryoballoon or Radiofrequency Ablation for Paroxysmal Atrial Fibrillation. N Engl J Med. 2016; 374(23): 2235-45.

6. Kuck KH, Fürnkranz A, Chun KR, Fürnkranz A, Busch M, Elvan A, et al; FIRE AND ICE Investigators. Cryoballoon or radiofrequency ablation for symptomatic paroxysmal atrial fibrillation: Reintervention, rehospitalization, and quality-of-life outcomes in the FIRE and ICE trial. Eur Heart J. 2016; 37(38): 2858-65.

7. Hussein AA, Saliba WI, Martin DO, Bhargava M, Sherman M, Magnelli-Reyes C, et al. Natural history and long-term outcomes of ablated atrial fibrillation. Circ Arrhythm Electrophysiol. 2011;4:271-278.

8. Haissaguerre M, Jais P, Shah DC, Takahashi A, Hocini M, QuiniouG, et al. Spontaneous initiation of atrial fibrillation by ectopic beats originating in the pulmonary veins. $\mathrm{N}$ Engl $\mathrm{J}$ Med. 1998;339(10):659-66.

9. Verlato R, Pieragnoli P, lacopino S, Rauhe W, Molon G, Stabile G, et al. Cryoballoon or radiofrequency ablation? Alternating technique for repeat procedures in patients with atrial fibrillation. Pacing Clin Electrophysiol. 2020;43(7):687-697.

10. Gaztanaga L, Frankel DS, Kohari M, Kondapalli L, Zado ES, Marchlinski FE. Time to recurrence of atrial fibrillation influences outcome following catheter ablation. Heart Rhythm. 2013;10(1):2-9.

11. Ouyang F, Ernst S, Chun J, Bansch D, Li Y, Schaumann A, et al. Electrophysiological findings during ablation of persistent atrial fibrillation with electroanatomic mapping and double Lasso catheter technique. Circulation.2005;112(20):3038-48.

12. Cabrera JA, Ho SY, Climent V, Fuertes B, Murillo M, Sanchez-Quintana D. Morphological evidence of muscular connections between contiguous pulmonary venous orifices: relevance of the 
interpulmonary isthmus for catheter ablation in atrial fibrillation. Heart Rhythm. 2009;6:1192-1198.

13. Ho SY, Sanchez-Quintana D, Cabrera JA, Anderson RH. Anatomy of the left atrium:implications for radiofrequency ablation of atrial fibrillation. J Cardiovasc Electrophysiol.1999;10:1525-1533.

14. Rajappan K, Kistler PM, Earley MJ, Thomas G, Izquierdo M, Sporton SC, et al. Acute and chronic pulmonary vein reconnection after atrial fibrillation ablation:a prospective characterization of anatomical sites. Pacing Clin Electrophysiol. 2008;31:1598-1605.

15. McLellan AJ, Ling LH, Ruggiero D, Wong MC, Walters TE, Nisbet A, et al. Pulmonary vein isolation:the impact of pulmonary venous anatomy on long-term outcome of catheter ablation for paroxysmal atrial fibrillation. Heart Rhythm. 2014;11:549-556.

16. De Regibus V, lacopino S, Abugattas JP, Mugnai G, Moran D,Ströker E, et al. Repeat procedures using the second-generation cryoballoon for recurrence of atrial fibrillation after initial ablation with conventional radiofrequency. J Interv Card Electrophysiol. 2017;49:119-125.

17. Haines DE, Verow AF. Observations on electrode-tissue interface temperature and effect on electrical impedance during radiofrequency ablation of ventricular myocardium. Circulation. 1990; 82:1034-8.

18. Nath S, Haines DE. Biophysics and pathology of catheter energy delivery systems. Prog Cardiovasc Dis. 1995;37:185-204.

19. Haines DE, Watson DD. Tissue heating during radiofrequency catheter ablation: a thermodynamic model and observations in isolated perfused and superfused canine right ventricular free wall. Pacing Clin Electrophysiol. 1989;12:962-76.

20. Calkins H, Prystowsky E, Carlson M, Klein LS, Saul JP, Gillette P. Temperature monitoring during radiofrequency catheter ablation procedures using closed loop control. Atakr Multicenter Investigators Group. Circulation. 1994;90:1279-86.

21. Schmidt M, Marschang H, Clifford S, Harald R, Guido R, Oliver T,et al. Trends in inflammatory biomarkers during atrial fibrillation ablation across different catheter ablation strategies. Int $\mathrm{J}$ Cardiol.2012; 158: 33-38.

22. Galand V, Pavin D, Behar N, Auffret V, Fénéon D, Behaghel A, et al. Localization of gaps during redo ablations of paroxysmal atrial fibrillation: Preferential patterns depending on the choice of cryoballoon ablation or radiofrequency ablation for the initial procedure. Arch Cardiovasc Dis.2016;109(11):591-8.

23. Sanchez-Quintana D, Cabrera JA, Climent V, Farre J, Mendonca MC, Ho SY. Anatomic relations between the esophagus and left atrium and relevance for ablation of atrial fibrillation. Circulation. 2005;112:1400-1405.

24. Kistler PM, Earley MJ, Harris S, Abrams D, Ellis S, Sporton SC,et al. Validation of three-dimensional cardiac image integration: Use of integrated CT image into electroanatomic mapping system to perform catheter ablation of atrial fibrillation. J Cardiovasc Electrophysiol. 2006;17:341-348.

25. Pappone C, Rosanio S, Oreto G, Tocchi M, Gugliotta F, Vicedomini G, et al. Circumferential radiofrequency ablation of pulmonary vein ostia: A new anatomic approach for curing atrial fibrillation. Circulation. 2000;102:2619-2628.

Page $12 / 16$ 
26. Ciconte G, Velagić V, Mugnai G, Saitoh Y, Irfan G, Hunuk B, et al. Electrophysiological findings following pulmonary vein isolation using radiofrequency catheter guided by contact-force and secondgeneration cryoballoon: lessons from repeat ablation procedures. Europace. 2016;18:71-77.

27. Miao C, Ju W, Chen H, Yang G, Zhang F, Gu K, et al. Clinical and electrophysiological characteristics predicting the re-ablation outcome for atrial fibrillation patients. J Interv Card Electrophysiol. 2020;59:373-379.

\section{Tables}

Due to technical limitations, tables are only available as a download in the Supplemental Files section.

\section{Figures}
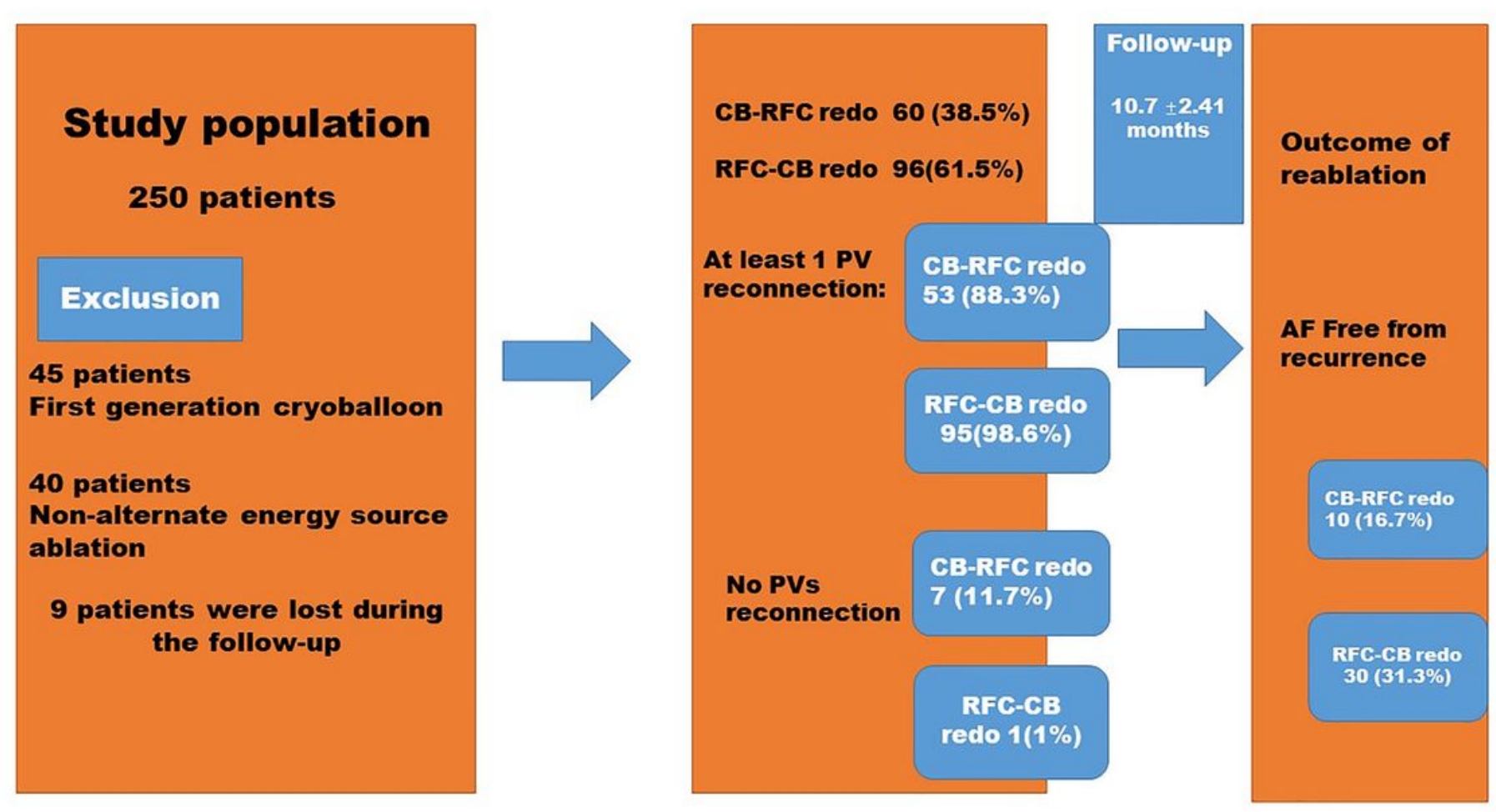

\section{Figure 1}

Research flow chart 


\section{0}

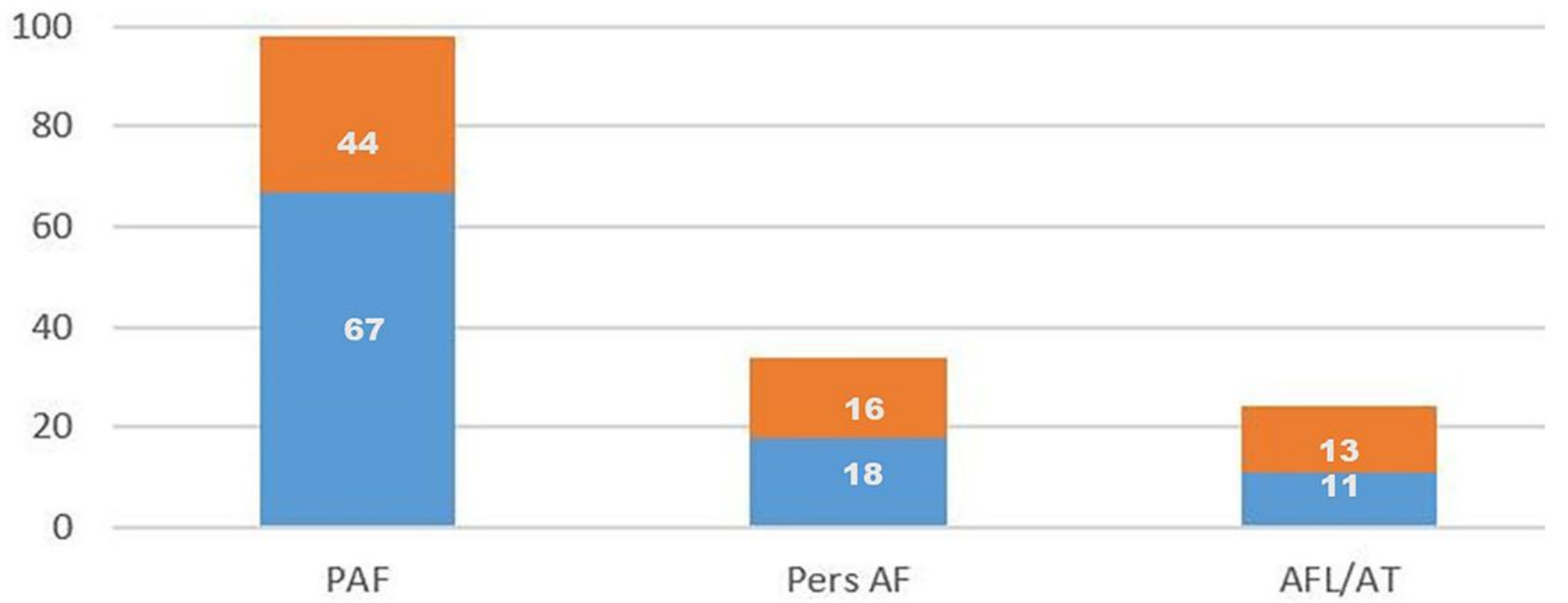

RFC-CB-redo $\quad$ CB-RFC-redo

\section{Figure 2}

Recurrent arrhythmias before re-ablation. Paroxysmal atrial fibrillation (PAF) was the most prevalent recurrent arrhythmia in both patient groups (RFC-CB redo group vs CB-RFC redo group). Numbers in stacked columns denote numbers of patients. AFL indicates atrial flutter; AT indicates atrial tachycardia; PAF, paroxysmal atrial fibrillation; PersAF, persistent atrial fibrillation.

A

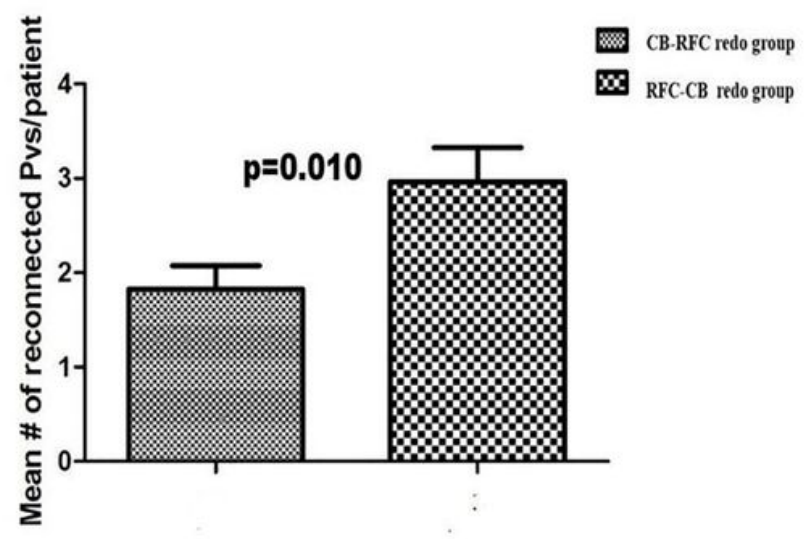

B

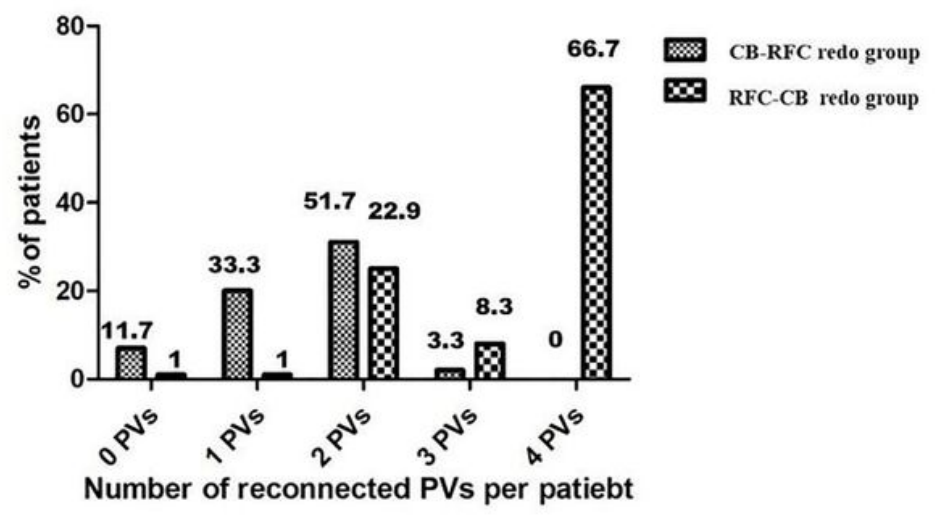

Figure 3 
Pulmonary vein (PV) reconnections identified during repeat ablation. A, Mean number of reconnected PVs according to patient group. B, Percentage of patients with 0 to 4 reconnected PVs per patient according to patient group.

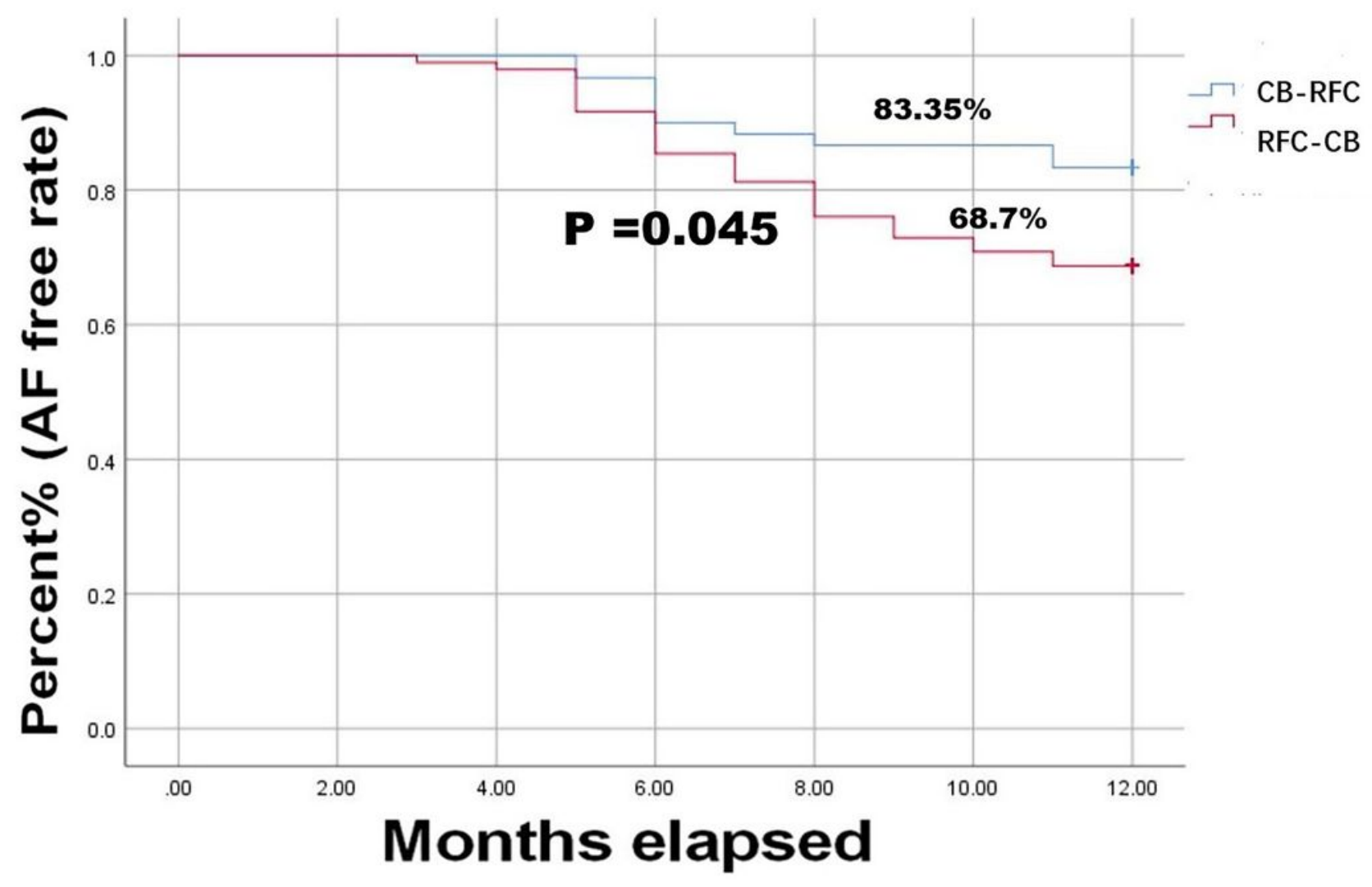

Figure 4

Freedom from recurrent atrial fibrillation (AF) following the repeat catheter ablation. RFC-CB-redo group: patients firstly ablated with RFC ablation underwent re-ablation with CB; CB- RFC-redo group: patients initially treated with $\mathrm{CB}$ underwent redo PV isolation with RFC ablation. 
A

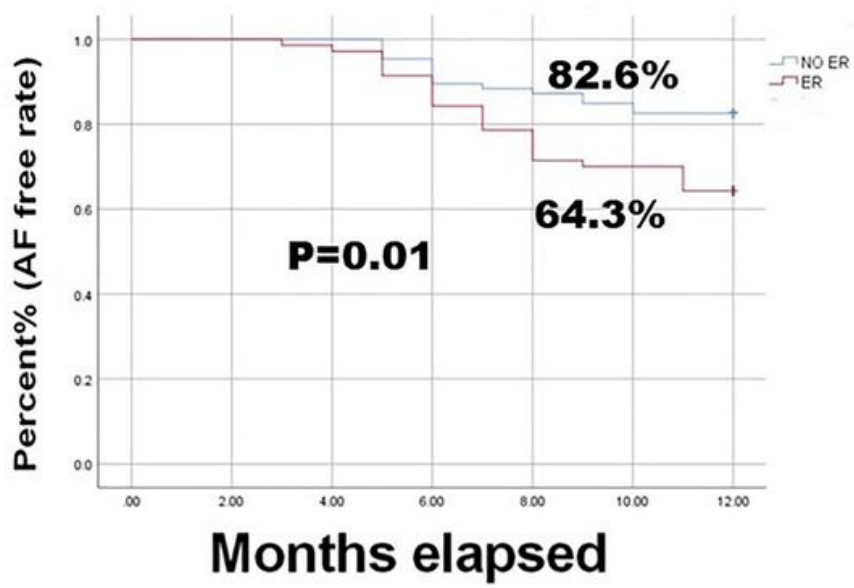

B

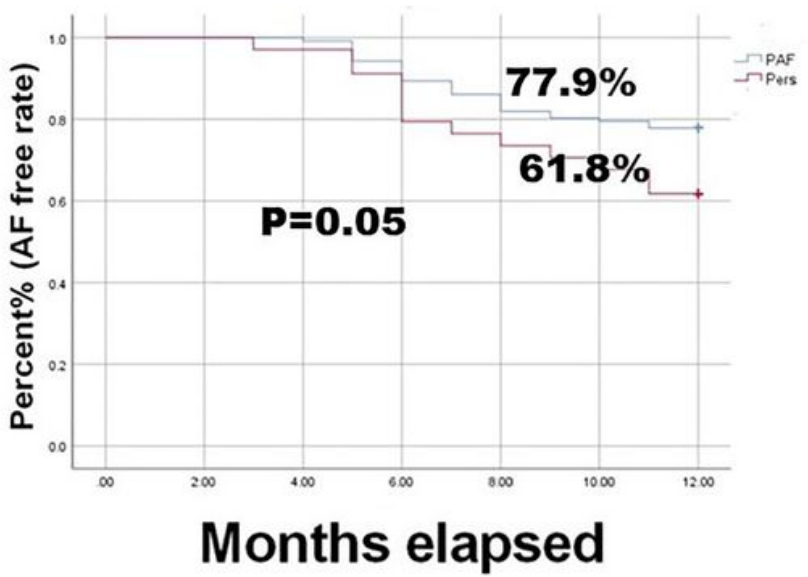

Figure 5

Subgroup analysis of freedom from recurrent atrial fibrillation (AF) following the repeat catheter ablation. A, early recurrence (ER) after the initial ablation vs no early recurrence (ER) after the initial ablation; $B, P A F$, paroxysmal atrial fibrillation vs PersAF, persistent atrial fibrillation.

\section{Supplementary Files}

This is a list of supplementary files associated with this preprint. Click to download.

- Table1.docx

- Table2.docx

- Table3.docx

- Table4.docx

- Table5.docx 